\title{
MAFLD is Associated with the Risk of Liver Fibrosis and Inflammatory Activity in $\mathrm{HBeAg}-\mathrm{Negative} \mathrm{CHB}$ Patients
}

\author{
Xiaoman Chen $\mathbb{D}^{1,2, *}$, Jing Zhou ${ }^{3, *}$, Lili Wu', Xiang Zhu', Hong Deng' \\ 'Department of Infectious Diseases, Third Affiliated Hospital of Sun Yat-sen University, Guangzhou, People's Republic of China; ${ }^{2}$ Infectious Disease \\ Center, Guangzhou Eighth People's Hospital, Guangzhou Medical University, Guangzhou, People's Republic of China; ${ }^{3}$ Department of Pathology, Third \\ Affiliated Hospital of Sun Yat-sen University, Guangzhou, People's Republic of China
}

*These authors contributed equally to this work

Correspondence: Hong Deng; Xiang Zhu, Department of Infectious Diseases, Third Affiliated Hospital of Sun Yat-sen University, No. 600 Tianhe Road, Tianhe District, Guangzhou, People's Republic of China, Tel +86-2085252506, Fax +86-2085252063, Email dhong@mail.sysu.edu.cn; zhuxiang@mail.sysu.edu.cn

Purpose: Chronic hepatitis B (CHB) and metabolic associated fatty liver disease (MAFLD) are both important public health problems. The effect of concomitant MAFLD on patients with CHB is still unclear. This study aimed to explore the influence of MAFLD on liver fibrosis and inflammation in CHB patients with different hepatitis B e antigen (HBeAg) status.

Patients and Methods: We retrospectively collected the clinical data of 399 treatment-naïve CHB patients who underwent liver biopsy. All patients were divided into two groups ( $\mathrm{HBeAg} \pm$ group). Logistic regression analysis was used to identify factors associated with liver inflammatory activity and significant fibrosis in patients with CHB. Multivariable logistic regressions were repeated in subgroups stratified by HBeAg status.

Results: In patients with CHB, MAFLD was independently associated with a risk of moderate-to-severe liver activity and significant fibrosis $(P<0.05)$. In the HBeAg-negative group, patients with MAFLD had significantly higher levels of alanine aminotransferase (ALT) $(P<0.05)$ and more severe liver inflammatory activity and fibrosis $(P<0.05)$ compared to those without MAFLD. MAFLD was independently associated with a risk of moderate-to-severe liver activity (A $\geq 3$ : OR 3.97, 95\% CI 1.71-9.22, $P=0.001)$ and significant fibrosis ( $\mathrm{F} \geq 2$ : OR 2.02, 95\% CI 1.09-3.73, $P=0.026$ ). In the HBeAg-positive group, MAFLD was found to be independently associated with moderate-to-severe liver activity (OR 2.44, 95\% CI 1.03-5.79, $P=0.044)$ but not fibrosis $(P=0.618)$.

Conclusion: MAFLD is associated with the risk of liver fibrosis and inflammatory activity in $\mathrm{HBeAg-negative} \mathrm{CHB} \mathrm{patients.}$ Sufficient attention should be paid to the prevention and treatment of MAFLD in patients with CHB, especially in HBeAg-negative patients.

Keywords: chronic hepatitis B, metabolic associated fatty liver disease, nonalcoholic fatty liver disease, hepatitis B e antigen

\section{Plain Language Summary}

Metabolic associated fatty liver disease (MAFLD) is a newly defined chronic liver disease, which refers to the presence of fatty liver and metabolic dysfunction. It affects a third of the global population. Chronic hepatitis B (CHB) is the main cause of liver-related mortality in Asia. In patients with $\mathrm{CHB}$, hepatitis B e antigen ( $\mathrm{HBeAg}$ ) is closely related to the risk of adverse outcomes. To better understand the influence of MAFLD on liver fibrosis and inflammation in CHB patients with different HBeAg status, we used logistic regression analysis to identify factors associated with liver inflammatory activity and significant fibrosis in patients with CHB. In HBeAg-negative patients, MAFLD is independently associated with a risk of moderate-to-severe liver activity and significant fibrosis. But in HBeAg-positive patients, MAFLD is found to be independently associated only with moderate-to-severe liver activity. MAFLD is associated with the risk of liver fibrosis and inflammatory activity in $\mathrm{HBe} A g$-negative $\mathrm{CHB}$ patients. It may aggravate liver damage in HBeAg-negative CHB patients. Sufficient attention should be paid to the prevention and treatment of MAFLD in patients with CHB, especially in HBeAg-negative patients. 


\section{Introduction}

Chronic hepatitis B (CHB) is a serious threat to human health. It can lead to decompensated liver cirrhosis, hepatocellular carcinoma, and even death. ${ }^{1}$ More than 250 million people worldwide suffered from chronic hepatitis B virus (HBV) infection and 887,000 died from cirrhosis and liver cancer in 2015, according to the World Health Organization (WHO) report. ${ }^{2}$ China is one of the countries with the highest burden of HBV infection. ${ }^{3}$ Many virological parameters are closely related to the risk of adverse outcomes, like HBV DNA quantification and hepatitis B e antigen ( $\mathrm{HBeAg})$ status. ${ }^{4-6}$ $\mathrm{HBeAg}$ is a protein secreted by $\mathrm{HBV}$-infected hepatocytes. It is an important immunomodulator in chronic $\mathrm{HBV}$ infection and is closely related to the immune tolerance of HBV in CHB patients. ${ }^{7-9}$ CHB patients with different $\mathrm{HBeAg}$ status have significant differences in viral replication and disease severity. ${ }^{10-12}$

Metabolic associated fatty liver disease (MAFLD) is the new definition of the previous nonalcoholic fatty liver disease (NAFLD) and refers to the presence of fatty liver and one or more of the metabolic risk factors (overweight/ obese, type 2 diabetes mellitus, metabolic dysregulation). ${ }^{13}$ MAFLD is an important global public health problem. It affects a third of the global population. ${ }^{14}$ MAFLD is associated with adverse outcomes such as cardiovascular events, hepatocellular cancer, and mortality. ${ }^{15-21}$

In recent years, with the improvement of living standards, the prevalence of CHB patients combined with MAFLD has increased incrementally, the epidemiologic data worldwide of which is lacking. A recent study conducted in China reported that the prevalence of MAFLD among patients with CHB is up to $32.2 \%(366 / 1135){ }^{22}$ The interaction between CHB and MAFLD has received increasing attention. Previous studies were mostly based on the old definition of "NAFLD", and the results are contradictory. Some researchers believed that NAFLD aggravates liver damage in patients with $\mathrm{CHB}$, ultimately promoting the occurrence of liver cirrhosis and liver cancer. ${ }^{23-25}$, However, other researchers believed that liver steatosis may inhibit HBV replication, facilitate HBsAg clearance, and reduce the risk of cirrhosis and human hepatocellular carcinoma (HCC) in patients with CHB. ${ }^{26-28}$ These "contradictory" conclusions confuse doctors and patients. This study aimed to explore the influence of MAFLD on liver fibrosis and inflammation in patients with $\mathrm{HBeAg}$-positive and $\mathrm{HBeAg}$-negative $\mathrm{CHB}$, thus providing a basis for the formulation of health management strategies for patients with CHB and MAFLD.

\section{Materials and Methods}

\section{Study Population}

This study retrospectively enrolled patients with CHB who had undergone liver biopsy at the Third Affiliated Hospital of Sun Yat-Sen University between December 2009 and August 2020 without receiving antiviral therapy. The inclusion criteria were as follows: 1) 18-65 years old; 2) HBsAg positive for at least 6 months; and 3) underwent liver biopsy before August 2020. The exclusion criteria were: 1) HBsAg negative before the biopsy; 2) Combined with the following liver diseases: other viral hepatitis, alcoholic liver disease, inherited liver disease, drug-induced liver injury, liver cancer; 3) Combined with severe acute infection; 4) Received anti-hepatitis B virus treatment before; 5) long-term treatment with antihypertensive, hypoglycemic or lipid-lowering drugs. Studies were conducted in accordance with the Declaration of Helsinki. Ethical approval was given by the medical ethics committee of the Third Affiliated Hospital of Sun Yat-Sen University, and informed consent was waived because we used de-identified retrospective data (Approval number: zssy[2020]02-191-01).

\section{Data Collection}

All subjects had complete data. Demographic data included age, gender, height, weight, body mass index (BMI), blood pressure, history of alcohol consumption, disease, and medication. Biochemical data were obtained prior to biopsy on the day of biopsy, including serum aspartate aminotransferase (AST), alanine aminotransferase (ALT), alkaline phosphatase (ALP), gamma-glutamyl transpeptidase (GGT), total bile acid (TBA), cholinesterase (CHE), fasting blood glucose (FBG), triacylglycerol (TRIG), high-density lipoprotein cholesterol (HDL), low-density lipoprotein cholesterol (LDL), creatinine (CREAT), and uric acid (UA). Viral data were collected from their most recent results before biopsy within 6 months, including serum HBV DNA level and the HBeAg status. 
The biopsy data were obtained from the Pathology Department. An experienced pathologist blinded to the clinical data reread the liver biopsy tissue with hematoxylin-eosin staining and reticulated fiber dyeing. Liver steatosis, inflammation and fibrosis were reported according to the Nonalcoholic Steatohepatitis Clinical Research Network (NASH CRN) and Scheuer scoring system. ${ }^{29,30}$

\section{Definitions and Criteria}

In this study, MAFLD was defined as at least 5\% liver steatosis pathologically, and the patients had one of the following metabolic risks: 1) overweight or obese (BMI $\left.\geq 23 \mathrm{~kg} / \mathrm{m}^{2}\right)$; 2) type 2 diabetes mellitus (T2DM); and 3) evidence of metabolic dysregulation. ${ }^{17}$ Metabolic dysregulation was defined as the presence of two or more of the following conditions: 1) high blood pressure (HBP): blood pressure $\geq 130 / 85 \mathrm{mmHg}$ or hypotensive drug treatment; 2) TRIG $\geq 1.70 \mathrm{mmol} / \mathrm{L}$ or lipid-lowering drug treatment; 3) HDL-c $<1.0 \mathrm{mmol} / \mathrm{L}$ for males and $<1.3 \mathrm{mmol} / \mathrm{L}$ for females or lipid-regulating drug treatment; and 4) prediabetes. Three additional proposed criteria for metabolic dysregulation (waist circumference, Homeostasis Model Assessment index and high sensitivity $\mathrm{C}$ reactive proteins) were not available in the medical records.

CHB was defined as the persistent presence of serum HBV surface antigen (HBsAg) for $>6$ months. Patients with MAFLD and CHB (MAFLD-CHB) were defined as patients fulfilling the criteria for both MAFLD and CHB. NonMAFLD-CHB was defined as patients who met the diagnostic criteria for CHB but did not meet the diagnostic criteria for MAFLD.

The liver activity score (A) was defined as the sum of the lobular inflammation score and the ballooning score. Moderate-to-severe liver activity was defined as a liver activity score $(A) \geq 3$. Significant fibrosis was defined as fibrosis staging of the NASH CRN system $(F) \geq 2$.

\section{Statistical Analysis}

All statistical analyses were performed with SPSS 25.5. Continuous variables are expressed as medians and interquartile ranges, and categorical variables are expressed as numbers and percentages. The Wilcoxon rank-sum test was used for continuous variables, and the chi-squared test was used for categorical variables. Logistic regression analysis was used to determine the relationship between MAFLD and liver pathological injury ( $\mathrm{A} \geq 3$ and $\mathrm{F} \geq 2$ ). The variance inflation factor (VIF) was used to evaluate collinearity; features with VIF $>10$ were excluded. Variables with $P<0.15$ in the univariate analysis were included in the multivariate analysis. A two-tailed $P$ value $<0.05$ was considered statistically significant.

Subgroup analyses: Multivariable logistic regressions were repeated to assess potential differential effects of MAFLD on liver inflammation and fibrosis in HBeAg-negative and HBeAg-positive subgroups. $P$-values for differences in associations among patients with $\mathrm{HBeAg-positive} \mathrm{and} \mathrm{HBeAg}$-negative $\mathrm{CHB}$ were calculated with 2 -sample t-tests based on the estimated changes and standard errors, and $P$-value $<0.05$ was considered statistically significant.

\section{Results}

\section{Baseline Characteristic}

The baseline characteristics were shown in supplementary materials (Tables S1 and S2). A total of 399 patients with CHB were enrolled, including $219 \mathrm{HBeAg}$-negative patients and $180 \mathrm{HBeAg}$-positive patients. MAFLD was diagnosed in $102(25.6 \%)$ participants. Compared with HBeAg-positive patients, HBeAg-negative patients had less liver inflammatory activity (A $\geq 3: 37$ [16.9] vs 47 [26.1], $P=0.025 ; \mathrm{G} \geq 2$ : 103 [47.0] vs 107 [59.4], $P=0.013$ ), and no significant differences in liver steatosis or fibrosis were found between HBeAg-positive patients and HBeAg-negative patients $(P>0.05)$. 


\section{Comparison Between MAFLD-CHB and Non-MAFLD-CHB in HBeAg-Positive and $\mathrm{HBeAg-Negative} \mathrm{Patients}$}

As shown in Tables 1 and 2, compared with the non-MAFLD-CHB patients, patients with MAFLD-CHB had a significantly higher proportion of males and a higher prevalence of HBP. Irrespective of their HBeAg status, patients with MAFLD-CHB had significantly higher levels of GGT, CHE, CREAT, UA and TRIG, but lower levels of HDL (all $P<0.05)$. The median age and HBV DNA quantitation between MAFLD-CHB patients and non-MAFLD-CHB patients were not significantly different $(P>0.05)$.

In the HBeAg-negative group, the levels of ALT (38 (27-48) vs 32 (23-41) U/L, $P=0.016)$ and FBG (5.25 (4.93$6.28)$ vs $5.12(4.68-5.47) \mathrm{mmol} / \mathrm{L}, P=0.011)$ were significantly higher in MAFLD-CHB patients than non-MAFLD-CHB patients. In the HBeAg-positive group, however, there were no significant differences in the above indicators between MAFLD-CHB patients and non-MAFLD-CHB patients $(P>0.05)$.

In the HBeAg-negative group, the liver activity score and fibrosis based on the NASH CRN system were more severe in patients with MAFLD-CHB than those with non-MAFLD-CHB (Figure 1). MAFLD-CHB patients had higher proportions of moderate-to-severe liver activity $(\mathrm{A} \geq 3: 21$ [32.8] vs 16 [10.3], $P<0.001)$ and significant fibrosis $(\mathrm{F} \geq 2: 41$ [64.1] vs 72 [46.5], $P=0.018)$ than non-MAFLD-CHB patients. In the HBeAg-positive group, the liver activity score based on the NASH CRN system was more severe in patients with MAFLD-CHB than those with non-MAFLD-CHB $(P=0.004)$, but no significant differences in liver lobular inflammation or fibrosis were observed $(P>0.05)$. Regardless of HBeAg status, no pathological differences were observed in liver inflammation or fibrosis between MAFLD-CHB and Non-MAFLD-CHB patients based on the Scheuer scoring system $(P>0.05)$.

Table I Comparison of Clinical Characteristics of CHB with and without MAFLD, Stratified by HBeAg Status

\begin{tabular}{|l|c|c|c|c|c|}
\hline & HBeAg- & & & \multicolumn{3}{|c|}{ HBeAg+ } \\
\cline { 2 - 6 } & $\begin{array}{c}\text { Non-MAFLD-CHB } \\
\text { (n=l55) }\end{array}$ & $\begin{array}{c}\text { MAFLD-CHB } \\
(\mathbf{n}=64)\end{array}$ & $\boldsymbol{P}$ & $\begin{array}{c}\text { Non-MAFLD-CHB } \\
(\mathbf{n = 1 4 2})\end{array}$ & MAFLD-CHB \\
$(\mathbf{n = 3 8 )}$
\end{tabular}

Notes: All data are expressed as medians (interquartile range), or $\mathrm{n}[\%]$, as appropriate; $P$-values $<0.05$ were considered statistically significant and have been bolded; NonMAFLD-CHB, defined as patients who met the diagnostic criteria for CHB but did not meet the diagnostic criteria for MAFLD; MAFLD-CHB, defined as patients fulfilling the criteria for both MAFLD and CHB.

Abbreviations: $\mathrm{CHB}$, chronic hepatitis B; MAFLD, metabolic associated fatty liver disease; $\mathrm{HBeAg}$, hepatitis B e antigen; T2DM, type 2 diabetes mellitus; BMI, body mass index; HBP, high blood pressure; AST, serum aspartate aminotransferase; ALT, alanine aminotransferase; GGT, gamma-glutamyl transpeptidase; ALP, alkaline phosphatase; TBA, total bile acid; CHE, cholinesterase, FBG, fasting blood glucose; CREAT, creatinine; UA, uric acid; TRIG, triacylglycerol; HDL-c, high-density lipoprotein cholesterol; LDL, low-density lipoprotein cholesterol. 
Table 2 Comparison of Pathological Characteristics of CHB with and without MAFLD, Stratified by HBeAg Status

\begin{tabular}{|c|c|c|c|c|c|c|}
\hline & \multicolumn{3}{|c|}{ HBeAg- } & \multicolumn{3}{|c|}{ HBeAg+ } \\
\hline & $\begin{array}{c}\text { Non-MAFLD-CHB } \\
(n=155)\end{array}$ & $\begin{array}{l}\text { MAFLD-CHB } \\
\quad(n=64)\end{array}$ & $P$ & $\begin{array}{l}\text { Non-MAFLD-CHB } \\
(n=142)\end{array}$ & $\begin{array}{l}\text { MAFLD-CHB } \\
\qquad(n=38)\end{array}$ & $P$ \\
\hline NASH CRN & & & & & & \\
\hline Steatosis & & & $<0.001$ & & & $<0.001$ \\
\hline so & $149[96.1]$ & $0[0.0]$ & & $135[95.1]$ & $0[0.0]$ & \\
\hline SI & 6[3.9] & $53[82.8]$ & & $7[4.9]$ & $30[78.9]$ & \\
\hline S23 & $0[0.0]$ & $\mathrm{II}[\mathrm{I7.2]}$ & & $0[0.0]$ & $8[21.1]$ & \\
\hline Lobular & & & 0.023 & & & 0.126 \\
\hline inflammation & & & & & & \\
\hline 0 & $24[15.5]$ & $3[4.7]$ & & $18[12.7]$ & $\mathrm{I}[2.6]$ & \\
\hline I & $10 \mid[65.2]$ & $39[60.9]$ & & $70[49.3]$ & $19[50.0]$ & \\
\hline 2 & $2 \mathrm{I}[13.5]$ & $14[21.9]$ & & $3 \mid[2 \mid .8]$ & $13[34.2]$ & \\
\hline 3 & $9[5.8]$ & $8[12.5]$ & & $23[16.2]$ & $5[13.2]$ & \\
\hline Ballooning & & & $<0.001$ & & & $<0.001$ \\
\hline 0 & $129[83.2]$ & $20[31.3]$ & & I24[87.3] & $15[39.5]$ & \\
\hline 1 & $21[13.5]$ & $39[60.9]$ & & $16[11.3]$ & $19[50.0]$ & \\
\hline 2 & $5[3.2]$ & $5[7.8]$ & & $2[1.4]$ & $4[10.5]$ & \\
\hline Activity score & & & $<0.001$ & & & 0.004 \\
\hline $\mathrm{A} \geq 3$ & $16[10.3]$ & $2 \mathrm{I}[32.8]$ & $<0.001$ & $30[21.1]$ & I7[44.7] & 0.003 \\
\hline$A \geq 4$ & $6[3.9]$ & $7[10.9]$ & 0.089 & $5[3.5]$ & $5[13.2]$ & 0.057 \\
\hline Fibrosis stage & & & 0.006 & & & 0.615 \\
\hline F0 & $16[10.3]$ & $4[6.3]$ & & $15[10.6]$ & $\mathrm{I}[2.6]$ & \\
\hline $\mathrm{FI}$ & $67[43.2]$ & $19[29.7]$ & & $6 I[43.0]$ & $16[42.1]$ & \\
\hline $\mathrm{F} 2$ & $28[18.1]$ & $26[40.6]$ & & $33[23.2]$ & $\mathrm{II}[28.9]$ & \\
\hline F3 & $23[14.8]$ & $\mathrm{II}[\mathrm{I7.2]}$ & & $19[13.4]$ & $6[15.8]$ & \\
\hline F4 & $2 \mid[13.5]$ & $4[6.3]$ & & |4[9.9] & $4[10.5]$ & \\
\hline $\mathrm{F} \geq 2$ & $72[46.5]$ & $4 I[64.1]$ & 0.018 & $33[23.2]$ & $10[26.3]$ & 0.693 \\
\hline Scheuer & & & & & & \\
\hline Activity grade & & & 0.201 & & & 0.579 \\
\hline G0I & $85[54.8]$ & $3 \mid[48.4]$ & & $56[39.4]$ & I7[44.7] & \\
\hline $\mathrm{G} 2$ & $43[27.7]$ & $23[35.9]$ & & $53[37.3]$ & $13[34.2]$ & \\
\hline G3 & $13[8.4]$ & $8[12.5]$ & & $22[15.5]$ & $7[18.4]$ & \\
\hline G4 & $14[9.0]$ & $2[3.1]$ & & $\mathrm{II}[7.7]$ & $\mathrm{I}[2.6]$ & \\
\hline Fibrosis stage & & & 0.538 & & & 0.276 \\
\hline $\mathrm{S}^{\prime} 0$ & $16[10.3]$ & $5[7.8]$ & & $16[11.3]$ & $\mathrm{I}[2.6]$ & \\
\hline$S^{\prime} I$ & $30[19.4]$ & $13[20.3]$ & & $33[23.2]$ & $14[36.8]$ & \\
\hline$S^{\prime} 2$ & $67[43.2]$ & $3 \mid[48.4]$ & & $60[42.3]$ & $13[34.2]$ & \\
\hline$S^{\prime} 3$ & $2 \mid[13.5]$ & $\mathrm{II}[17.2]$ & & $19[13.4]$ & $6[15.8]$ & \\
\hline$S^{\prime} 4$ & $21[13.5]$ & $4[6.3]$ & & |4[9.9] & $4[10.5]$ & \\
\hline
\end{tabular}

Notes: All data are expressed as $\mathrm{n}$ [\%]; $P$-values $<0.05$ were considered statistically significant and have been bolded; Non-MAFLD-CHB, defined as patients who met the diagnostic criteria for $\mathrm{CHB}$ but did not meet the diagnostic criteria for MAFLD; MAFLD-CHB, defined as patients fulfilling the criteria for both MAFLD and CHB.

Abbreviations: CHB, chronic hepatitis B; MAFLD, metabolic associated fatty liver disease; HBeAg, hepatitis B e antigen; NASH CRN, the Nonalcoholic Steatohepatitis Clinical Research Network.

\section{MAFLD Was Associated with Liver Inflammatory Activity and Fibrosis}

Effect of MAFLD on the Risk of Moderate-to-Severe Liver Activity $(A \geq 3)$ in Patients with CHB

In univariate analysis, combined MAFLD, BMI $\geq 23 \mathrm{~kg} / \mathrm{m} 2$, prediabetes, HBeAg positivity, and HBV DNA quantitative $>6$ $\log 10 \mathrm{IU} / \mathrm{mL}$ were significantly associated with the risk of moderate-to-severe liver activity $(P<0.05)($ Table 3$)$. In multivariate analysis, after adjusting for the influence of HBeAg, gender, BMI, and prediabetes, combined with MAFLD (OR, 3.06; 95\% CI, 1.69-5.54, $P<0.001)$ and HBV DNA quantitative $>6 \log 10 \mathrm{IU} / \mathrm{mL}(\mathrm{OR}, 2.40 ; 95 \% \mathrm{CI}, 1.18-4.88, P=0.015)$ were independently associated with the risk of moderate-to-severe liver activity. In the subgroup analysis, regardless of 

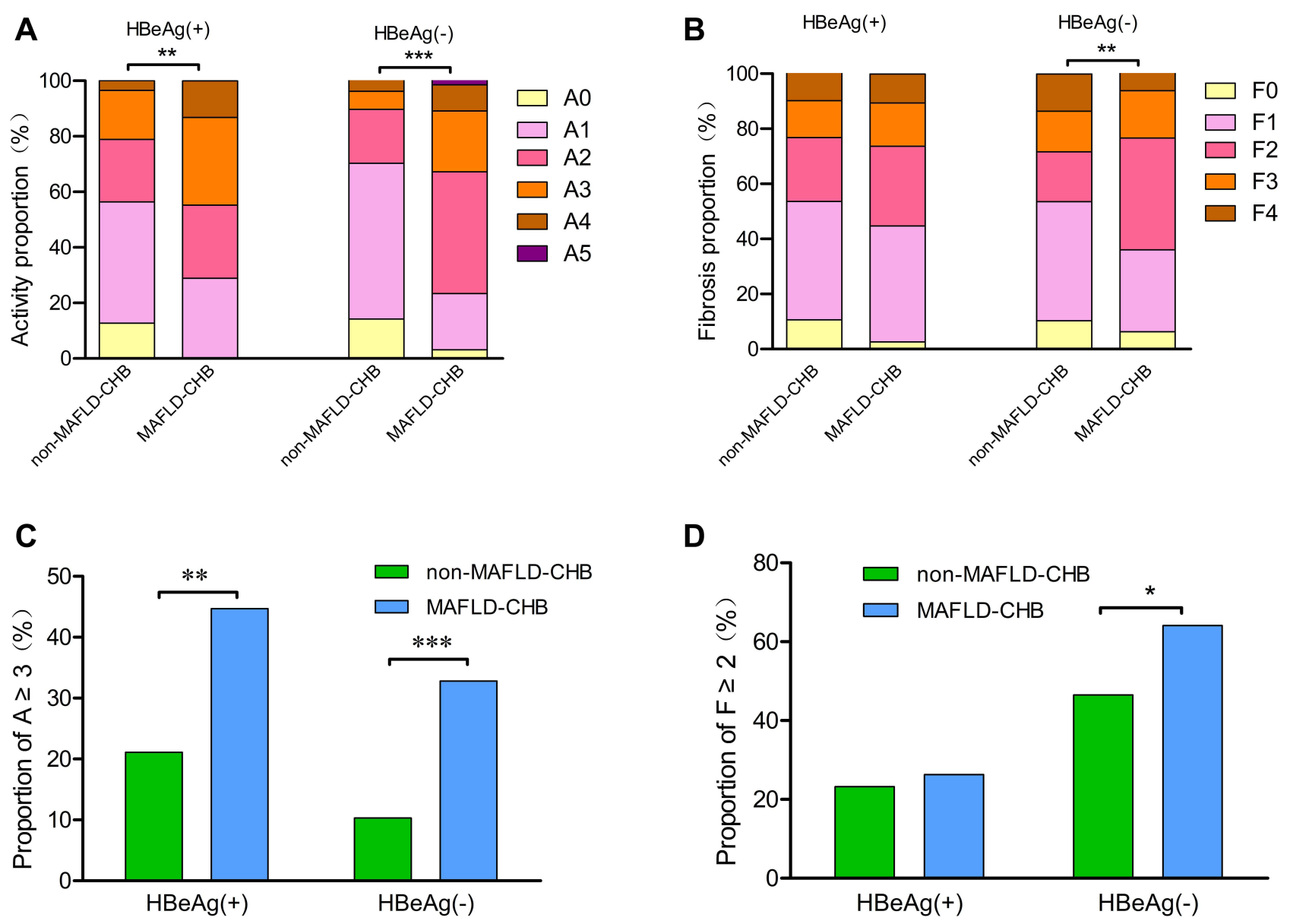

Figure I Comparison of liver activity score and fibrosis stage distributions between MAFLD-CHB and non-MAFLD-CHB groups under the NASH CRN system, stratified by $\mathrm{HBeAg}$ status (A) Comparison of liver activity score distribution; (B) Comparison of liver fibrosis stage distribution; (C) Comparison of proportion of $A$ :3; (D) Comparison of proportion of $\mathrm{F} \geq 2$; MAFLD-CHB, defined as patients fulfilling the criteria for both MAFLD and CHB; Non-MAFLD-CHB, defined as patients who met the diagnostic criteria for CHB but did not meet the diagnostic criteria for MAFLD.

Abbreviations: MAFLD, metabolic associated fatty liver disease; CHB, chronic hepatitis B; NASH CRN, the Nonalcoholic Steatohepatitis Clinical Research Network; $\mathrm{HBeAg}$, hepatitis $\mathrm{B}$ e antigen; $\mathrm{A}$, liver activity score; F, fibrosis stages; ${ }^{*} p<0.05$, ${ }^{* *} p<0.0 \mathrm{I}$, and $*^{* *} p<0.00 \mathrm{I}$.

HBeAg status, MAFLD was independently associated with the risk of moderate-to-severe liver activity $(P<0.05)$, while HBV DNA quantitation was only independently associated with the risk of moderate-to-severe liver activity in the HBeAg-negative subgroup (Table 4). No significant difference was detected between subgroups stratified by HBeAg status $(P>0.05)$.

\section{Effect of MAFLD on the Risk of Significant Fibrosis $(F \geq 2)$ in Patients with CHB}

In univariate analysis, male and MAFLD were significantly associated with the risk of significant fibrosis $(P<0.05)$, and HBeAg and age were not significantly associated with liver fibrosis $(P>0.05)$ (Table 3$)$. In multivariate analysis, after adjusting for the influence of gender, HBeAg and HBV DNA quantitation, MAFLD was independently associated with the risk of significant fibrosis (OR, 1.64; 95\% CI, 1.02-2.63; $P=0.040)$. In the subgroup analysis, MAFLD was still independently associated with significant fibrosis in the HBeAg-negative subgroup (OR, 2.02; 95\% CI, 1.09-3.73; $P=0.026)$ but not in the HBeAg-positive subgroup $(P>0.05)$ (Table 5). In the HBeAg-positive subgroup, HBV DNA quantitative $>6 \log 10 \mathrm{IU} /$ $\mathrm{mL}$ were independently associated with the lower risk of significant fibrosis (OR, $0.31 ; 95 \% \mathrm{CI}, 0.15-0.64 ; P=0.002)$. The effect of HBV DNA on liver fibrosis was significantly different between subgroups stratified by $\mathrm{HBeAg}$ status $(P=0.001)$.

\section{Discussion}

This study explored the impact of MAFLD on liver fibrosis and inflammation in patients with HBeAg-positive and HBeAg-negative CHB. We demonstrated that MAFLD was associated with the risk of liver fibrosis and inflammatory 
Table 3 Univariate Analysis of Factors Associated with Moderate-to-Severe Liver Activity $(A \geq 3)$ and Significant Fibrosis $(F \geq 2)$ in Patients with $\mathrm{CHB}$

\begin{tabular}{|c|c|c|c|c|}
\hline \multirow[t]{2}{*}{ Variates } & \multicolumn{2}{|c|}{ Moderate-to-Severe Liver Activity $(A \geq 3)$} & \multicolumn{2}{|c|}{ Significant Fibrosis (F $\geq 2$ ) } \\
\hline & OR $(95 \% \mathrm{Cl})$ & $\mathbf{P}$ & OR $(95 \% \mathrm{CI})$ & $\mathbf{P}$ \\
\hline Male & $0.63(0.35-1.12)$ & 0.115 & $1.68(1.07-2.63)$ & 0.023 \\
\hline Age & $0.99(0.96-1.02)$ & 0.391 & $1.00(0.98-1.03)$ & 0.802 \\
\hline MAFLD & $3.24(1.95-5.39)$ & $<0.001$ & $1.84(1.19-2.87)$ & 0.007 \\
\hline T2DM & $0.53(0.06-4.37)$ & 0.555 & $3.05(0.6 I-15.28)$ & 0.176 \\
\hline $\mathrm{BMI} \geq 23 \mathrm{~kg} / \mathrm{m}^{2}$ & $1.87(1.13-3.09)$ & 0.015 & $1.24(0.83-1.83)$ & 0.293 \\
\hline HBP & $1.05(0.58-1.89)$ & 0.873 & $0.91(0.56-1.47)$ & 0.692 \\
\hline Hypertriglyceridemia & $0.98(0.54-1.78)$ & 0.936 & $0.83(0.51-1.34)$ & 0.442 \\
\hline Low HDL cholesterolemia & $1.29(0.79-2.13)$ & 0.309 & $1.04(0.69-1.57)$ & 0.862 \\
\hline Prediabetes & $1.76(1.02-3.03)$ & 0.043 & $1.34(0.83-2.16)$ & 0.234 \\
\hline HBV DNA quantitative $>6 \log 10 \mathrm{IU} / \mathrm{mL}$ & $2.31(1.42-3.76)$ & $<0.001$ & $0.72(0.48-1.08)$ & 0.114 \\
\hline $\mathrm{HBeAg}(+)$ & $1.74(1.07-2.82)$ & 0.026 & $0.88(0.59-1.30)$ & 0.516 \\
\hline
\end{tabular}

Notes: Data were expressed as odds ratios (OR) and $95 \%$ confidence interval (Cl); $P$-values $<0.15$ were considered statistically significant and have been bolded; Variables with $P<0.15$ in the univariate analysis were included in the multivariate analysis; All variables of variance inflation factor (VIF: 1.037-2.018) were less than 5-10; Hypertriglyceridemia, triacylglycerol (TRIG) $\geq 1.70 \mathrm{mmol} / \mathrm{L}$; Low HDL cholesterolemia, HDL-c $<1.0 \mathrm{mmol} / \mathrm{L}$ for males and $<1.3 \mathrm{mmol} / \mathrm{L}$ for females.

Abbreviations: $\mathrm{CHB}$, chronic hepatitis B; OR, odds ratio; MAFLD, metabolic associated fatty liver disease; T2DM, type 2 diabetes mellitus; BMI, Body mass index; HBP, high blood pressure; $\mathrm{HBeAg}$, hepatitis $\mathrm{B}$ e antigen; VIF, variance inflation factor; TRIG, triacylglycerol; HDL-c, high-density lipoprotein cholesterol.

Table 4 Multivariate Analysis of Factors Associated with Moderate-to-Severe Liver Activity (A $\geq 3)$ in Patients with CHB and Stratified by $\mathrm{HBeAg}$ Status

\begin{tabular}{|c|c|c|c|c|c|c|c|}
\hline \multirow[t]{2}{*}{ Variates } & \multicolumn{2}{|l|}{ Total } & \multirow{2}{*}{$\begin{array}{c}\text { HBeAg (-) } \\
\text { OR (95\% Cl) }\end{array}$} & \multirow[b]{2}{*}{$P$} & \multicolumn{2}{|c|}{ HBeAg (+) } & \multirow[b]{2}{*}{$P^{a}$} \\
\hline & OR $(95 \% \mathrm{Cl})$ & $\boldsymbol{P}$ & & & OR $(95 \% \mathrm{CI})$ & $P$ & \\
\hline $\mathrm{HBeAg}(+)$ & $1.18(0.58-2.4 \mathrm{I})$ & 0.656 & & & & & \\
\hline Male & $1.19(0.63-2.24)$ & 0.597 & $0.79(0.3 \mathrm{I}-2.04)$ & 0.631 & $1.53(0.64-3.65)$ & 0.339 & 0.311 \\
\hline HBV DNA quantitative $>6 \log 10 \mathrm{IU} / \mathrm{mL}$ & $2.40(1.18-4.88)$ & 0.015 & $4.44(1.49-13.19)$ & 0.007 & $1.59(0.68-3.72)$ & 0.280 & 0.146 \\
\hline $\mathrm{BMI} \geq 23 \mathrm{~kg} / \mathrm{m}^{2}$ & $1.23(0.69-2.21)$ & 0.489 & $1.25(0.52-3.00)$ & 0.612 & $\mathrm{I} .26(0.56-2.8 \mathrm{I})$ & 0.581 & 0.990 \\
\hline Prediabetes & $1.58(0.88-2.84)$ & 0.128 & $2.33(1.04-5.25)$ & 0.041 & $0.99(0.4-2.44)$ & 0.987 & 0.167 \\
\hline MAFLD & $3.06(1.69-5.54)$ & $<0.001$ & $3.97(1.71-9.22)$ & 0.001 & $2.44(1.03-5.79)$ & 0.044 & 0.429 \\
\hline Constant & 0.08 & $<0.001$ & 0.08 & $<0.001$ & 0.13 & $<0.001$ & \\
\hline
\end{tabular}

Notes: Data were expressed as odds ratios (OR) and $95 \%$ confidence interval (CI); All variables of variance inflation factor (VIF: I.054-I.903) were less than 5-I0; ${ }^{2} P$-values for difference in associations among $\mathrm{HBeAg}$ positive and $\mathrm{HBeAg}$ negative were calculated with 2-sample t-tests based on the estimated changes and standard errors; $P$-values $<0.05$ were considered statistically significant and have been bolded.

Abbreviations: $\mathrm{CHB}$, chronic hepatitis B; HBeAg, hepatitis B e antigen; OR, odds ratio; MAFLD, metabolic associated fatty liver disease; BMI, Body mass index; VIF, variance inflation factor.

Table 5 Multivariate Analysis of Factors Associated with Significant Fibrosis (F $\geq 2)$ in Patients with CHB and Stratified by HBeAg Status

\begin{tabular}{|c|c|c|c|c|c|c|c|}
\hline \multirow[t]{2}{*}{ Variates } & \multicolumn{2}{|c|}{ Total } & \multicolumn{2}{|c|}{ HBeAg (-) } & \multicolumn{2}{|c|}{ HBeAg (+) } & \multirow[b]{2}{*}{$P^{a}$} \\
\hline & OR $(95 \% \mathrm{CI})$ & $\boldsymbol{P}$ & OR $(95 \% \mathrm{CI})$ & $P$ & OR $(95 \% \mathrm{CI})$ & $\boldsymbol{P}$ & \\
\hline $\mathrm{HBeAg}(+)$ & $1.22(0.7-2.12)$ & 0.482 & & & & & \\
\hline Male & $1.49(0.94-2.36)$ & 0.093 & $1.13(0.60-2.12)$ & 0.698 & $2.13(1.04-4.36)$ & 0.038 & 0.194 \\
\hline HBV DNA quantitative $>6 \log 10 \mathrm{lU} / \mathrm{mL}$ & $0.66(0.37-1.15)$ & 0.143 & $2.91(1.00-8.49)$ & 0.051 & $0.31(0.15-0.64)$ & 0.002 & 0.001 \\
\hline MAFLD & $1.64(1.02-2.63)$ & 0.040 & $2.02(1.09-3.73)$ & 0.026 & $\mathrm{I} .22(0.57-2.6 \mathrm{I})$ & 0.618 & 0.313 \\
\hline Constant & 0.72 & 0.134 & 0.73 & 0.260 & 1.28 & 0.566 & \\
\hline
\end{tabular}

Notes: Data were expressed as odds ratios (OR) and 95\% confidence interval (Cl); All variables of variance inflation factor (VIF: I.054-I.885) were less than 5-I0; ${ }^{\text {P }}$-values for difference in associations among $\mathrm{HBeAg}$ positive and $\mathrm{HBeAg}$ negative were calculated with 2-sample t-tests based on the estimated changes and standard errors; $P$-values $<0.05$ were considered statistically significant and have been bolded.

Abbreviations: $\mathrm{CHB}$, chronic hepatitis $\mathrm{B} ; \mathrm{HBeAg}$, hepatitis $\mathrm{B}$ e antigen; OR, odds ratio; MAFLD, metabolic associated fatty liver disease; VIF, variance inflation factor. 
activity in HBeAg-negative CHB patients. In our study, MAFLD was independently associated with the risk of moderateto-severe liver activity and significant fibrosis $(P<0.05)$. In the HBeAg-negative subgroup, MAFLD was independently associated with the risk of moderate-to-severe liver activity and significant fibrosis. However, in the HBeAg-positive subgroup, MAFLD was found to be independently associated only with moderate-to-severe liver activity. We also found no significant association between MAFLD and virological indicators such as HBV DNA quantification or HBeAg status.

The new definition of MAFLD allows the comorbid conditions of MAFLD and CHB. ${ }^{13}$ We reported that CHB patients combined with MAFLD had more severe liver pathological damage than those without MAFLD. Few studies have analyzed the role of MAFLD in the disease severity of CHB. A study in Toronto and Netherlands established a retrospective cohort of biopsy-proven CHB and found that the presence of MAFLD in CHB patients was associated with liver-related adverse outcomes. ${ }^{31}$ However, another study in Taiwan reported no relationship between the overall prognosis in HBV-related HCC and the presence of MAFLD. ${ }^{32}$ More studies were based on the old definitions of "NAFLD". NAFLD is defined as excess fat accumulation (at least 5\%) in the liver of patients without a history of alcohol abuse or other causes of secondary hepatic steatosis. Nonalcoholic steatohepatitis (NASH) is considered the severe stage of NAFLD, defined as hepatic steatosis with liver inflammation and hepatocyte death. ${ }^{33}$ It's been found to be associated with metabolic syndrome, ${ }^{24}$ which is similar to our findings (Table S8-S11). Previous studies have reported that NASH is associated with severe liver fibrosis and adverse liver-related outcomes. ${ }^{24,34}$ However, none of the above studies reported an independent correlation between NAFLD and liver fibrosis or adverse outcomes. Differences in the definitions of those fatty liver diseases, study populations, and study methods may account for the inconsistent results of different studies. In addition, the evaluation of liver lesions in those studies were different, some of them lacked pathological confirmation, and the others used different pathological scoring systems. In the analysis of Scheuer scoring system, we found no association between MAFLD and the risk of liver fibrosis in patients with CHB (Tables 2 and $\underline{\mathrm{S} 2}$ ). However, in the analysis of the NASH CRN system, we found a prominent difference. Scheuer scoring system tended to underestimate early fibrosis in our study (Table S7). We believe that the evaluation of liver fibrosis in CHB with MAFLD cannot be based solely on the traditional CHB scoring system, and the combination of the NASH CRN system may be a more appropriate choice.

In the subgroup analysis, we reported that MAFLD was only associated with the risk of liver fibrosis in patients with HBeAg-negative CHB, but not in those with HBeAg-positive CHB. The effect of MAFLD on the risk of liver fibrosis appeared more pronounced in patients with $\mathrm{HBeAg}$-negative $\mathrm{CHB}$, but the $P$-value for group difference was not statistically significant $(P=0.313)$. Studies on the relationship between fatty liver disease and fibrosis under different HBeAg status are still rare. Previous studies indicated that liver steatosis was associated with the risk of elevated ALT, liver fibrosis, and fibrosis progression in HBeAg-negative CHB patients, implying a poor prognosis. ${ }^{35,36}$ Few studies discussed the relationship between $\mathrm{HBeAg}$-positive CHB and fatty liver disease. Only one retrospective study found that in HBeAg-positive patients, the proportion of liver cirrhosis was significantly higher in patients with liver steatosis, but no significant difference was found after matching for age and sex. ${ }^{37}$ Similarly, we also found that MAFLD was not significantly associated with liver fibrosis in HBeAg-positive patients, indicating that the effect of MAFLD on HBeAgpositive patients is not dramatic.

In the present study, high HBV DNA load was found to be independently associated with liver inflammatory activity. Previous studies have also reported that high HBV DNA load was associated with adverse liver outcomes (HCC, liverrelated death). ${ }^{4,38}$ Notably, in our study, high HBV DNA load was associated with the increased risk of significant liver fibrosis in the total population of $\mathrm{CHB}$, but with the decreased risk of liver fibrosis in patients with HBeAg-positive CHB. A study in Australia also found that the influence of HBV DNA levels differed in HBeAg-positive and HBeAg-negative patients. ${ }^{39}$ This can be explained by the natural history of CHB. ${ }^{40}$

This study revealed several novel findings about the influence of MAFLD on liver fibrosis and inflammation in patients with $\mathrm{CHB}$, and few studies have reported before. We re-reviewed the pathology slides of CHB patients and applied two pathological scoring systems to evaluate the degree of liver injury. Besides, we conducted a pre-specified subgroup analysis stratified by $\mathrm{HBeAg}$ status in an attempt to provide more accurate medical advice in different populations. MAFLD may aggravate liver damage in patients with $\mathrm{CHB}$, especially in those with HBeAg-negative 
CHB. Interventions to improve MAFLD in patients with CHB are necessary, especially in patients with HBeAg-negative CHB, who may benefit more from such interventions.

However, this study also has limitations. As a retrospective study, the data obtained lack information about the waist circumference, Homeostasis Model assessment index, and high sensitivity $\mathrm{C}$ reactive proteins, so the definition of metabolic dysfunction in this study did not include these three indicators. The prevalence of MAFLD may have been potentially underestimated, but underestimation should be limited. In our study group, 13 of 399 patients met criteria for at least 5\% liver steatosis but did not meet criteria for MAFLD. Some of these 13 patients may indeed have MAFLD. To explore whether this limitation would have affected our main results, repeated analyses were performed substituting fatty liver disease (at least 5\% liver steatosis pathologically) for MAFLD (Tables S3-6). The main findings did not change. Additional large-sample prospective studies with fulfilling criteria of MAFLD are needed to further clarify the relationship between MAFLD and CHB.

\section{Conclusion}

In summary, MAFLD is associated with the risk of liver fibrosis and inflammatory activity in HBeAg-negative CHB patients. Sufficient attention should be paid to the prevention and treatment of MAFLD in patients with CHB, especially in HBeAg-negative patients.

\section{Abbreviations}

CHB, chronic hepatitis B; MAFLD, metabolic associated fatty liver disease; HBeAg, hepatitis B e antigen; HBV, chronic hepatitis B virus; WHO, the World Health Organization; NAFLD, nonalcoholic fatty liver disease; HCC, human hepatocellular carcinoma; BMI, Body mass index; AST, serum aspartate aminotransferase; ALT, alanine aminotransferase; ALP, alkaline phosphatase; GGT, gamma-glutamyl transpeptidase; TBA, total bile acid; CHE, cholinesterase, FBG, fasting blood glucose; TRIG, triacylglycerol; HDL-c, high-density lipoprotein cholesterol; LDL, low-density lipoprotein cholesterol; Cr, creatinine; UA, uric acid; NASH CRN, the Nonalcoholic Steatohepatitis Clinical Research Network; T2DM, type 2 diabetes mellitus; HBP, high blood pressure; HBsAg, HBV surface antigen; VIF, variance inflation factor; NASH, Nonalcoholic steatohepatitis; OR, odds ratio.

\section{Data Sharing Statement}

The data used to support the findings of this study have not been made available because no patient approval has been obtained.

\section{Ethics Approval and Informed Consent}

Studies were conducted in accordance with the Declaration of Helsinki. Ethical approval was given by the medical ethics committee of the Third Affiliated Hospital of Sun Yat-Sen University, and informed consent was waived because we used de-identified retrospective data (Approval number: zssy[2020]02-191-01).

\section{Consent for Publication}

All authors gave final approval of the version to be published and agreed to be listed as authors.

\section{Acknowledgments}

We acknowledge Yunting Zhang for her help in statistics. We also gratefully acknowledge our study group members for their work.

\section{Author Contributions}

Xiaoman Chen, study design, acquisition of data, analysis, drafted and written; Jing Zhou, study design, pathologic evaluation, analysis, drafted; Lili Wu, acquisition of data, analysis, drafted; Xiang Zhu, study design, analysis, reviewed and revised the draft; Hong Deng, study design, analysis, reviewed and revised the draft. All authors contributed to data 
analysis, drafting, or revising the article, have agreed on the journal to which the article will be submitted, gave final approval of the version to be published, and agree to be accountable for all aspects of the work.

\section{Funding}

This work was supported by grants from the National Natural Science Foundation of China [grant number 81870597].

\section{Disclosure}

Dr Xiaoman Chen reports grants from the National Natural Science Foundation of China, during the conduct of the study. The authors declare that there are no conflicts of interest regarding the publication of this article.

\section{References}

1. Yang JD, Gyedu A, Afihene MY, et al. Hepatocellular carcinoma occurs at an earlier age in africans, particularly in association with chronic Hepatitis B. Am J Gastroenterol. 2015;110(11):1629-1631. doi:10.1038/ajg.2015.289

2. World Health Organization. Global hepatitis report, 2017; 2017. Available from: https://www.who.int/hepatitis/publications/global-hepatitisreport2017/en/. Accessed April 2017

3. Chinese national administration of disease prevention and control. Overview of the Chinese notifiable infectious diseases; 2018-2020. Available from: http://www.nhc.gov.cn/jkj/pgzdt/new_list.shtml. Accessed January 24, 2022.

4. Chen CJ, Yang HI, Su J, et al. Risk of hepatocellular carcinoma across a biological gradient of serum hepatitis B virus DNA level. JAMA. 2006;295 (1):65-73.

5. Yang H-I, Lu S-N, Liaw Y-F, et al. Hepatitis B e antigen and the risk of hepatocellular carcinoma. N Engl J Med. 2002;347(3):168-174. doi:10.1056/NEJMoa013215

6. Tseng TC, Liu CJ, Hsu CY, et al. High level of Hepatitis B core-related antigen associated with increased risk of hepatocellular carcinoma in patients with chronic HBV infection of intermediate viral load. Gastroenterology. 2019;157(6):1518-1529 e1513. doi:10.1053/j. gastro.2019.08.028

7. Will H. HBcAg and HBeAg expression: how where, why or why not?. J Hepatol. 1991;13(Suppl 4):S66-S67.

8. Kramvis A, Kostaki EG, Hatzakis A, Paraskevis D. Immunomodulatory function of HBeAg related to short-sighted evolution, transmissibility, and clinical manifestation of Hepatitis B virus. Front Microbiol. 2018;9:2521. doi:10.3389/fmicb.2018.02521

9. Milich D, Liang TJ. Exploring the biological basis of hepatitis B e antigen in hepatitis B virus infection. Hepatology. 2003;38(5):1075-1086. doi:10.1053/jhep.2003.50453

10. Wang J, Wu W, Yan X, et al. HBeAg negativity is associated with more advanced liver fibrosis in patients with chronic Hepatitis B: a propensity score-matching analysis. J Clin Gastroenterol. 2020;54(9):826-831. doi:10.1097/MCG.0000000000001291

11. Bai H, Liu H, Chen X, Xu C, Dou X. Influence of age and HBeAg status on the correlation between HBV DNA and hepatic inflammation and fibrosis in chronic hepatitis B patients. Dig Dis Sci. 2013;58(5):1355-1362. doi:10.1007/s10620-012-2479-7

12. Hui CK, Leung N, Yuen ST, et al. Natural history and disease progression in Chinese chronic hepatitis B patients in immune-tolerant phase. Hepatology. 2007;46(2):395-401. doi:10.1002/hep.21724

13. Eslam M, Sarin SK, Wong VW, et al. The Asian Pacific association for the study of the liver clinical practice guidelines for the diagnosis and management of metabolic associated fatty liver disease. Hepatol Int. 2020;14(6):889-919. doi:10.1007/s12072-020-10094-2

14. Lin S, Huang J, Wang M, et al. Comparison of MAFLD and NAFLD diagnostic criteria in real world. Liver Int. 2020;40(9):2082-2089. doi:10.1111/liv.14548

15. Lee H, Lee Y-H, Kim SU, Kim HC. Metabolic dysfunction-associated fatty liver disease and incident cardiovascular disease risk: a Nationwide Cohort Study. Clin gastroenterol hepatol. 2021;19(10):2138-2147.e10. doi:10.1016/j.cgh.2020.12.022

16. Tsutsumi T, Eslam M, Kawaguchi T, et al. MAFLD better predicts the progression of atherosclerotic cardiovascular risk than NAFLD: generalized estimating equation approach. Hepatol Res. 2021;51(11):1115-1128. doi:10.1111/hepr.13685

17. Guerreiro GTS, Longo L, Fonseca MA, de Souza VEG, Álvares-da-silva MR. Does the risk of cardiovascular events differ between biopsy-proven NAFLD and MAFLD? Hepatol Int. 2021;15(2):380-391. doi:10.1007/s12072-021-10157-y

18. Vitale A, Svegliati-Baroni G, Ortolani A, et al. Epidemiological trends and trajectories of MAFLD-associated hepatocellular carcinoma 2002-2033: the ITA.LI.CA database. Gut;2021. gutjnl-2021-324915. doi:10.1136/gutjnl-2021-324915

19. Myers S, Neyroud-Caspar I, Spahr L, et al. NAFLD and MAFLD as emerging causes of HCC: a populational study. JHEP Rep. $2021 ; 3(2): 100231$. doi:10.1016/j.jhepr.2021.100231

20. Wang X, Wu S, Yuan X, et al. Metabolic dysfunction-associated fatty liver disease and mortality among chinese adults: a Prospective Cohort Study. $J$ Clin Endocrinol Metab. 2022;107(2):e745-e755. doi:10.1210/clinem/dgab644

21. Huang Q, Zou X, Wen X, Zhou X, Ji L. NAFLD or MAFLD: which has closer association with all-cause and cause-specific mortality?-Results from NHANES III. Front Med. 2021;8:693507. doi:10.3389/fmed.2021.693507

22. Huang J, Xue W, Wang M, et al. MAFLD criteria may overlook a subtype of patient with steatohepatitis and significant fibrosis. Diabetes Metab Syndr Obes. 2021;14:3417-3425. doi:10.2147/DMSO.S316096

23. Lee YB, Ha Y, Chon YE, et al. Association between hepatic steatosis and the development of hepatocellular carcinoma in patients with chronic hepatitis B. Clin Mol Hepatol. 2019;25(1):52-64. doi:10.3350/cmh.2018.0040

24. Charatcharoenwitthaya P, Pongpaibul A, Kaosombatwattana U, et al. The prevalence of steatohepatitis in chronic hepatitis B patients and its impact on disease severity and treatment response. Liver Int. 2017;37(4):542-551. doi:10.1111/liv.13271

25. Fan JG, Chen GF, Ji D, et al. Long-term disease progression and prevalence of metabolic syndrome in chronic hepatitis B patients with comorbid nonalcoholic fatty liver disease. Hepatology. 2016;64:538A-538A. 
26. Hu D, Wang H, Wang H, et al. Non-alcoholic hepatic steatosis attenuates hepatitis B virus replication in an HBV-immunocompetent mouse model. Hepatol Int. 2018;12(5):438-446. doi:10.1007/s12072-018-9877-7

27. Hui RWH, Seto WK, Cheung KS, et al. Inverse relationship between hepatic steatosis and hepatitis B viremia: results of a large case-control study. $J$ Viral Hepat. 2018;25(1):97-104. doi:10.1111/jvh.12766

28. Li J, Yang HI, Yeh ML, et al. Association between fatty liver and cirrhosis, hepatocellular carcinoma, and HBsAg seroclearance in chronic hepatitis B. J Infect Dis. 2020;4:54.

29. Kleiner DE, Brunt EM, Van Natta M, et al. Design and validation of a histological scoring system for nonalcoholic fatty liver disease. Hepatology. 2005;41(6):1313-1321. doi:10.1002/hep.20701

30. Scheuer PJ. Classification of chronic viral hepatitis: a need for reassessment. J Hepatol. 1991;13(3):372-374. doi:10.1016/0168-8278(91)90084-O

31. Van kleef LA, Choi HSJ, Brouwer WP, et al. Metabolic dysfunction-associated fatty liver disease increases risk of adverse outcomes in patients with chronic hepatitis B. JHEP Rep. 2021;3(5):100350. doi:10.1016/j.jhepr.2021.100350

32. Lin YP, Lin SH, Wang CC, et al. Impact of MAFLD on HBV-related stage 0/A hepatocellular carcinoma after curative resection. $J$ Pers Med. 2021;11(8):684. doi:10.3390/jpm11080684

33. Chalasani N, Younossi Z, Lavine JE, et al. The diagnosis and management of non-alcoholic fatty liver disease: practice Guideline by the American Association for the Study of Liver Diseases, American College of Gastroenterology, and the American Gastroenterological Association. Hepatology. 2012;55(6):2005-2023. doi:10.1002/hep.25762

34. Choi HSJ, Brouwer WP, Zanjir WMR, et al. Nonalcoholic steatohepatitis is associated with liver-related outcomes and all-cause mortality in chronic Hepatitis B. Hepatology. 2020;71(2):539-548. doi:10.1002/hep.30857

35. Mak L-Y, Seto W-K, Hui RW-H, et al. Fibrosis evolution in chronic hepatitis B e antigen-negative patients across a 10-year interval. J Viral Hepat. 2019;26(7):818-827. doi:10.1111/jvh.13095

36. Enomoto H, Aizawa N, Nishikawa H, et al. Relationship between hepatic steatosis and the elevation of aminotransferases in HBV-infected patients With HBe-Antigen negativity and a low viral load. Medicine. 2016;95(17):e3565. doi:10.1097/MD.0000000000003565

37. Chen Y-C, Jeng W-J, Hsu C-W, Lin C-Y. Impact of hepatic steatosis on treatment response in nuclesos(t)ide analogue-treated HBeAg-positive chronic hepatitis B: a retrospective study. BMC Gastroenterol. 2020;20(1):146. doi:10.1186/s12876-020-01289-w

38. Fattovich G, Olivari N, Pasino M, D’Onofrio M, Martone E, Donato F. Long-term outcome of chronic hepatitis B in Caucasian patients: mortality after 25 years. Gut. 2008;57(1):84-90. doi:10.1136/gut.2007.128496

39. Croagh CM, Bell SJ, Slavin J, et al. Increasing hepatitis B viral load is associated with risk of significant liver fibrosis in HBeAg-negative but not HBeAg-positive chronic hepatitis B. Liver Int. 2010;30(8):1115-1122. doi:10.1111/j.1478-3231.2010.02267.x

40. Burns GS, Thompson AJ. Viral hepatitis B: clinical and epidemiological characteristics. Cold Spring Harb Perspect Med. 2014;4(12):a024935. doi:10.1101/cshperspect.a024935

Diabetes, Metabolic Syndrome and Obesity: Targets and Therapy

Dovepress

\section{Publish your work in this journal}

Diabetes, Metabolic Syndrome and Obesity: Targets and Therapy is an international, peer-reviewed open-access journal committed to the rapid publication of the latest laboratory and clinical findings in the fields of diabetes, metabolic syndrome and obesity research. Original research, review, case reports, hypothesis formation, expert opinion and commentaries are all considered for publication. The manuscript management system is completely online and includes a very quick and fair peer-review system, which is all easy to use. Visit http://www.dovepress. com/testimonials.php to read real quotes from published authors.

Submit your manuscript here: https://www.dovepress.com/diabetes-metabolic-syndrome-and-obesity-targets-and-therapy-journal 